\title{
Panel: Governance in Open Source Projects and Communities
}

\author{
Francesco Bolici ${ }^{1}$, Paul de Laat $^{2}$, Jan Ljungberg ${ }^{3}$, \\ Andrea Pontiggia ${ }^{4}$, and Cristina Rossi Lamastra ${ }^{5}$ \\ ${ }^{1}$ University of Cassino \\ ${ }^{2}$ University of Groningen \\ ${ }^{3}$ Göteborg University \\ ${ }^{4}$ Ca' Foscari University \\ ${ }^{5}$ Politecnico di Milano
}

\begin{abstract}
"Although considerable research has been devoted to the growth and expansion of open source communities and the comparison between the efficiency of corporate structures and community structures in the field of software development, rather less attention has been paid to their governance structures (control, monitoring, supervision)" (Lattemann and Stieglitz 2005).

The call for the identification and the description of a set of effective governance mechanisms in OSS development projects and community is still open and actual (Grandori 2000, de Laat 2007). The panel aims to discuss and to develop the recent ideas and contributions related to the governance structures inside OSS communities. Organizational, sociological, technical and psychological aspects will be considered as components of the same issue.

The main aim of the panel is to encourage discussion and exchange of ideas from different viewpoints. Particular attention will be dedicated to the discussion of the following topics: i) the identification of the range of governance tools actually in use within OSS; ii) how to coordinate the activities in OS projects: software modularity, task assignment and other emerging coordination structures for managing the developer's behaviour; iii) governance and design of the interactions between companies and OS communities; iv) copyleft: the needed prerequisite of the OSS model. We intend also to encourage the discussion about the possibility to apply the identified OSS governance mechanisms to other industries.
\end{abstract}

\section{References}

Lattemann, C., Stieglitz, S.: Framework for Governance in Open Source Communities. In: 38th Hawaii International Conference on Systems Sciences, Hawaii (2005)

Grandori, A.: Conjectures for a New Research Agenda on Governance. Journal of Management and Governance 4, 1-9 (2000)

de Laat, P.: Governance of open source software: state of the art. Journal of Management and Governance 11(2), 165-177 (2007) 